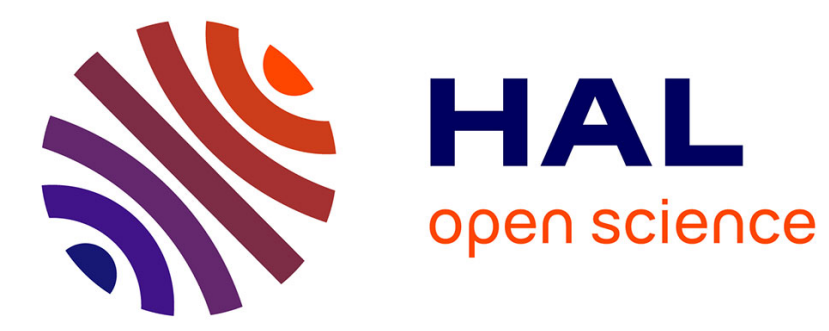

\title{
Analysis of ADCs by Native Mass Spectrometry
}

Oscar Hernandez-Alba, Anthony Ehkirch, Alain Beck, Sarah Cianférani

\section{To cite this version:}

Oscar Hernandez-Alba, Anthony Ehkirch, Alain Beck, Sarah Cianférani. Analysis of ADCs by Native Mass Spectrometry. L. Nathan Tumey. Antibody-Drug Conjugates, 2078, Springer, pp.197-211, 2020, Methods in Molecular Biology, 978-1-4939-9928-6. 10.1007/978-1-4939-9929-3_13 . hal-02341672

\section{HAL Id: hal-02341672 \\ https://hal.science/hal-02341672}

Submitted on 20 Oct 2020

HAL is a multi-disciplinary open access archive for the deposit and dissemination of scientific research documents, whether they are published or not. The documents may come from teaching and research institutions in France or abroad, or from public or private research centers.
L'archive ouverte pluridisciplinaire HAL, est destinée au dépôt et à la diffusion de documents scientifiques de niveau recherche, publiés ou non, émanant des établissements d'enseignement et de recherche français ou étrangers, des laboratoires publics ou privés. 


\section{Analysis of ADCs by Native Mass Spectrometry}

Oscar Hernandez-Alba ${ }^{1 *}$, Anthony Ehkirch ${ }^{1 *}$, Alain Beck ${ }^{2}$, Sarah Cianférani ${ }^{1}$

${ }^{1}$ Laboratoire de Spectrométrie de Masse Bio-Organique (LSMBO), IPHC, UMR 7178, Université de Strasbourg, CNRS, 25 rue Becquerel, 67087 Strasbourg, France

${ }^{2}$ Pierre Fabre Laboratories, IRPF - Centre d'Immunologie Pierre-Fabre (CIPF), 74160 SaintJulien-en-Genevois, France.

*These authors equally contributed to this work

\section{Corresponding author address:}

Sarah Cianférani, Laboratoire de Spectrométrie de Masse Bio-Organique (LSMBO), IPHC, UMR 7178, Université de Strasbourg, CNRS, 25 rue Becquerel, 67087 Strasbourg, France sarah.cianferani@unistra.fr 


\section{Running head}

Analysis of ADCs by native electrospray MS

\section{Summary/Abstract}

Mass spectrometry performed in non-denaturing conditions (native MS) has proven its utility for the quantitative and qualitative analysis of antibody-drug conjugates (ADCs), especially when ADCs' subunits involve non-covalent interactions (i.e. cysteine-conjugated ADCs). Its hyphenation to ion mobility spectroscopy (IM-MS) allows differentiation of gas-phase ions based on their rotationally averaged collision cross section providing an additional dimension of conformational characterization of ADCs. More recently, size exclusion chromatography (SEC) appeared as an interesting technique to perform online buffer exchange in an automated way prior to native MS/IM-MS analysis. Online SEC-native MS/IM-MS allows the global structural characterization of $\mathrm{ADCs}$ and the assessment of some critical quality attributes (CQAs) required for $\mathrm{ADC}$ release on the market, such as drug load distribution (DLD), drugto-antibody ratio (DAR), the average DAR (DAR $a v$ ), and the relative amount of unconjugated $\mathrm{mAb}$.

\section{Keywords}

Native mass spectrometry, Antibody drug conjugate, Ion mobility mass spectrometry, Size exclusion chromatography-native mass spectrometry 


\section{Introduction}

ADCs are tripartite molecules designed to improve the therapeutic efficacy of monoclonal antibodies (mAbs) through the covalent addition of a cytotoxic molecule via a linker[1]. The heterogeneity associated to ADCs can vary, depending on the bioconjugation chemistry used to attach the cytotoxic molecules to the mAb structure[2]. Several studies have shown the suitability of native mass spectrometry (MS) for ADC analysis[3-13]. The reduced charge state of the ions under non-denaturing conditions facilitates the identification and quantification of all the different DAR populations that can be potentially formed during the conjugation process. Furthermore, the rotationally averaged collision cross section (CCS) of each individual DAR population can be determined by using ion mobility coupled to mass spectrometry (IM-MS) to assess the impact of the conjugation process on the overall conformation of the original $\mathrm{mAb}[6$; 7]. However, sample preparation (desalting) is still mostly manual, labor intensive and timeconsuming prior to native IM-MS analysis. SEC-native MS not only allows on-line buffer exchange within few minutes but also provides separation, identification and quantification of high/low molecular weight species[9]. Online SEC-native MS can be easily implemented in high throughput automated environments thus pushing native MS approaches to the forefront of ADC characterization in R\&D laboratories.

\section{Materials}

All solutions are prepared with ultrapure water.

\subsection{Mobile phase for SEC-native MS}

$100 \mathrm{mM} \mathrm{AcONH}_{4}$, pH 6.8: weigh $3.08 \mathrm{~g}$ of $\mathrm{AcONH}_{4}$ in a $500 \mathrm{~mL}$ volumetric flask. Add $400 \mathrm{~mL}$ of milli-Q water with stirring. No $\mathrm{pH}$ adjustment is needed.

\subsection{Deglycosylated ADCs}


ADCs were obtained as European Union pharmaceutical-grade drug product from their respective manufacturers. The deglycosylation was performed by incubating for $30 \mathrm{~min}$ at $37^{\circ} \mathrm{C}$ one unit of IgGZERO (Genovis) per microgram of ADC.

\subsection{Manual buffer exchange}

1. $150 \mathrm{mM} \mathrm{AcONH}_{4}, \mathrm{pH}$ 6.8: weigh $578 \mathrm{mg}$ of $\mathrm{AcONH}_{4}$ in a $50 \mathrm{~mL}$ falcon. Add $50 \mathrm{~mL}$ of milli-Q water with stirring.

\subsection{Cesium Iodide solution for Mass Spectrometer calibration}

Prepare a $2 \mathrm{~g} / \mathrm{L}$ solution of cesium iodide in 50/50 isopropanol/water (v/v).

\section{a. Synapt G2}

1. The automated chip-based nanoelectrospray device (Triversa Nanomate, Advion, Ithaca, USA) is operated in the positive ion mode. Set the capillary voltage and the nitrogen nanoflow at $1.75 \mathrm{kV}$ and 0.60 psi respectively.

2. In MassLynx MS Tune windows, set the following parameters:

Operate the instrument in positive and sensitivity modes with a $\mathrm{m} / \mathrm{z}$ range from 1000 to 10000 .

Nanoflow + panel: Sampling Cone: 120 V. Extraction con: 5 V. Source Temperature: $90{ }^{\circ} \mathrm{C}$. The Capillary Voltage and the gas flows are not used in the Nanomate interface setup.

Instrument: Trap and Transfer Collision Energy are turned off.

3. Start a $2 \mathrm{~min}$ TOF-MS acquisition with a $\mathrm{m} / \mathrm{z}$ range from 1000 to 10000 .

4. Average the signal over the $2 \mathrm{~min}$ in the Chromatogram panel. In the spectrum panel, a Spectrum Smooth with \pm 10 Smooth window and a number of smooths of 2 with a "Mean" Smoothing method were realized. On the smooth spectrum, the TOF Spectrum Center with a min peak width at half height of 2 and a Centroid top at $80.00 \%$ was selected. 
5. In the Acquity UPLC Console, select "Create Calbration".

6. In the calibration profile editor, select the right Mass Calibration Profile (CsI in the $1000-10000 \mathrm{~m} / \mathrm{z}$ range in positive and sensitivity modes).

7. The Reference Compound (Csi_1000-10000 positive) with reference masses from 1172.1450 to $7927.2031 \mathrm{Da}$ ) is used for the calibration.

\section{b. Exactive Plus EMR}

1. Using HESI source and the $500 \mu \mathrm{L}$ Hamilton $^{\mathrm{TM}}$ syringe, deliver cesium iodide solution with a flow rate set to $10 \mu \mathrm{L} / \mathrm{min}$.

2. In the MS Tune page manually set the following parameters: Operate the instrument in positive mode with a m/z range from 1,000 to 20,000. Apply $25 \mathrm{eV}$ and $100 \mathrm{eV}$ for the $\mathrm{CID}$ and CE parameters, respectively. The source temperature is set at $250^{\circ} \mathrm{C}$, the capillary voltage is set to $4 \mathrm{kV}$ and the gas flows is set to 10 u.a. The trapping gas pressure is set to $7 \mathrm{u}$.a. The ion optics are set to $4 \mathrm{~V}$ for injection, inter and bent flatapoles. Set the nominal resolution at 17,500 and activate the EMR mode.

3. To perform the calibration, wait for a stable TIC $(<12 \%)$ then select "Calibrate" and check "EMR MS Mass Calibration (pos)".

\subsection{Ion mobility calibrants}

1. $10 \mathrm{mM} \mathrm{AcONH}_{4}$ : weight $38 \mu \mathrm{g}$ of $\mathrm{AcONH}_{4}$ in $50 \mathrm{~mL}$ falcon. Add $50 \mathrm{~mL}$ of mili-Q water with stirring.

2. Weigh between $400 \mu \mathrm{g}$ and $900 \mu \mathrm{g}$ of concanavalin A from canavalia ensiformis, alcohol dehydrogenase from saccharomyces cerevisiae, and pyruvate kinase from rabbit muscle. Dissolve each individual calibrant in $100 \mu \mathrm{L}$ of $10 \mathrm{mM}$ ammonium acetate to reach a final tetrameric concentration between $30-40 \mu \mathrm{M}$ (Note 1).

3. Perform manual buffer exchange using a $50 \mathrm{kD}$ microconcentrator (see 2.3. section). 
4. Use the nanodrop spectrophotometer to determine the concentration of the calibrant solutions after manual desalting.

5. Add the required volume of $10 \mathrm{mM}$ AcONH4 solution to get a final concentration of 10 $\mu \mathrm{M}$ for each individual calibrant solution.

\section{Methods}

\subsection{ADC sample preparation}

1. A microconcentrator with an appropriate MWCO (such as Vivaspin 50-kD cutoff for classical ADCs, Sartorius, Göttingen, Germany) is used for manual buffer exchange.

2. Equilibrate the microconcentrator by adding $500 \mu \mathrm{L}$ of $150 \mathrm{mM} \mathrm{AcONH}_{4}$ at $14000 \mathrm{~g}$ over 2 min and remove the eluate.

3. Repeat step 3 twice.

4. Load between $25-50 \mu \mathrm{L}$ of sample. Add $\mathrm{AcONH}_{4}$ buffer to get a final volume of 500 $\mu \mathrm{L}$.

5. Centrifuge for $10 \mathrm{~min}$ at $14000 \mathrm{~g}$ to concentrate down to $50 \mu \mathrm{L}$ and remove the eluate. Add $450 \mu \mathrm{L}$ of $\mathrm{AcONH}_{4}$ buffer. Repeat the dilution concentration cycle five times.

6. After the last cycle, transfer the $50 \mu \mathrm{L}$ of sample to an Eppendorf tube. Protein concentration is determined by UV absorbance using a NanoDrop spectrophotometer (Thermo Fisher Scientific, France).

\subsection{Mass spectrometer calibration}

\section{a. Synapt G2}

1. The automated chip-based nanoelectrospray device (Triversa Nanomate, Advion, Ithaca, USA) is operated in the positive ion mode. Set the capillary voltage and the nitrogen nanoflow at $1.75 \mathrm{kV}$ and 0.60 psi respectively. 
2. In MassLynx MS Tune windows, set the following parameters:

Operate the instrument in positive and sensitivity modes with a $\mathrm{m} / \mathrm{z}$ range from 1000 to 10000 .

Nanoflow+ panel: Sampling Cone: 120 V. Extraction con: 5 V. Source Temperature: $90{ }^{\circ} \mathrm{C}$. The Capillary Voltage and the gas flows are not used in the Nanomate interface setup.

Instrument: Trap and Transfer Collision Energy are turned off.

3. Start a $2 \mathrm{~min}$ TOF-MS acquisition with a m/z range from 1000 to 10000 .

4. Average the signal over the $2 \mathrm{~min}$ in the Chromatogram panel. In the spectrum panel, a Spectrum Smooth with \pm 10 Smooth window and a number of smooths of 2 with a "Mean" Smoothing method were realized. On the smooth spectrum, the TOF Spectrum Center with a min peak width at half height of 2 and a Centroid top at $80.00 \%$ was selected.

5. In the Acquity UPLC Console, select "Create Calbration".

6. In the calibration profile editor, select the right Mass Calibration Profile (CsI in the $1000-10000 \mathrm{~m} / \mathrm{z}$ range in positive and sensitivity modes).

7. The Reference Compound (Csi_1000-10000 positive) with reference masses from 1172.1450 to $7927.2031 \mathrm{Da})$ is used for the calibration.

\section{b. Exactive Plus EMR}

1. Using HESI source and the $500 \mu \mathrm{L}$ Hamilton $^{\mathrm{TM}}$ syringe, deliver cesium iodide solution with a flow rate set to $10 \mu \mathrm{L} / \mathrm{min}$.

2. In the MS Tune page manually set the following parameters: Operate the instrument in positive mode with a $\mathrm{m} / \mathrm{z}$ range from 1,000 to 20,000 . Apply $25 \mathrm{eV}$ and $100 \mathrm{eV}$ for the CID and CE parameters, respectively. The source temperature is set at $250^{\circ} \mathrm{C}$, the capillary voltage is set to $4 \mathrm{kV}$ and the gas flows is set to 10 u.a. The trapping gas 
pressure is set to 7 u.a. The ion optics are set to $4 \mathrm{~V}$ for injection, inter and bent flatapoles. Set the nominal resolution at 17,500 and activate the EMR mode.

3. To perform the calibration, wait for a stable TIC $(<12 \%)$ then select "Calibrate" and check "EMR MS Mass Calibration (pos)".

\subsection{Direct infusion on Exactive Plus EMR for native MS experiment}

\section{a. Mass spectrometry setting for native MS experiment}

1. Desalt between $100 \mu \mathrm{g}$ and $150 \mu \mathrm{g}$ of ADC in $150 \mathrm{mM}$ ammonium acetate.

2. Determine the concentration of the ADC solution after desalting by UV absorbance using NanoDrop spectrophotometer (Thermo Fisher Scientific, France).

3. Prior to ADC mass analysis, perform Exactive Plus EMR calibration with $8 \mu \mathrm{L}$ of cesium iodide solution (see 2.4).

4. Connect the automated chip-based nanoESI infusion source (Triversa Nanomate, Advion, Ithaca, USA) to the Exactive Plus EMR.

5. In the Tune page, set the following parameters: Positive mode with a $\mathrm{m} / \mathrm{z}$ range from 1 000 to 14000 . Activate the EMR mode and set the trapping gas pressure at 7 u.a., the ion optics at $8 \mathrm{~V}, 7 \mathrm{~V}, 6 \mathrm{~V}$ for inter, injection and bent flatapoles respectively, the S-lens $\mathrm{RF}$ voltage at $200 \mathrm{~V}$, the temperature at $250^{\circ} \mathrm{C}$, the fragmentation parameters at $75 \mathrm{eV}$ and $10 \mathrm{eV}$ for CID and CE values, respectively. Select a nominal resolution of 17500.

6. Start a 2 min acquisition with a stable TIC. The mass spectrum is presented in Figure 1 a.

\section{b. Data interpretation}

1. In BioPharma Finder 2.0, select your raw data file and Intact Protein Analysis.

2. In chromatogram \& Source Spectra, select a m/z range from 5500 to 7 500. In ReSpect Deconvolution Algorithm, set a Model Mass Range from 145000 to 160000 with a 
mass tolerance of $10 \mathrm{ppm}$. Select a charge state range from 18 to 35 . For the Choice of Peak Model, select Raw File Specific for the Resolution at 400 m/z.

3. Process the algorithm in order to have the deconvoluted spectrum presented in Figure $1 b$.

4. For average DAR (DARav), see 3.4. [Figure 1 near here]

\subsection{Direct infusion on Synapt G2 for native MS/IM-MS experiment}

\section{a. Mass spectrometry settings for native MS experiment}

1. Desalt between $100 \mu \mathrm{g}$ and $150 \mu \mathrm{g}$ of ADC in $150 \mathrm{mM}$ ammonium acetate.

2. Determine the concentration of the ADC solution after desalting by UV absorbance using NanoDrop spectrophotometer (Thermo Fisher Scientific, France).

3. Connect the automated chip-based nanoESI infusion source (Triversa Nanomate, Advion, Ithaca, USA) to the Synapt G2 HDMS (Waters, Manchester, UK).

4. The analysis on the Synapt G2 HDMS is performed in sensitive mode.

5. Prior to ADC mass analysis, perform Synapt $\mathrm{G} 2$ calibration with $8 \mu \mathrm{L}$ of cesium iodide solution (see 2.4).

6. In the MassLynx Tune windows set the following parameters: Positive ion mode, $\mathrm{m} / \mathrm{z}$ range from 1000 to 10000, sampling cone: $180 \mathrm{~V}$ (Note 2), backing pressure: 6 mbar, source offset: $5 \mathrm{~V}$, source temperature: $90^{\circ} \mathrm{C}$, trap collision energy: $10 \mathrm{~V}$, transfer collision energy: $5 \mathrm{~V}$, trap gas: $5 \mathrm{~mL} / \mathrm{min}$, scan time: $4 \mathrm{~s}$, and acquisition time: $2 \mathrm{~min}$.

7. Deliver among 10-20 $\mu \mathrm{L}$ of ADC solution at $5 \mu \mathrm{M}$ concentration.

8. In MaxEnt 1, the ranges are set from 145000 to 162000 with a resolution of 1 $\mathrm{Da} /$ channel. The width at half height is set to $2 \mathrm{Da}$ for the uniform Gaussian. The deconvoluted spectrum is presented in Figure $\mathbf{2 b}$.

9. For the DARav calculation, see 3.4.[Figure 2 near here]

\section{b. Data interpretation Synapt G2}


1. Open the chromatogram option in MassLynx menu.

2. Combine the mass signal over the two minutes acquisition time.

3. Smooth the signal using the following parameters: Smooth window: 10, number of smooths: 2, and smoothing method: Mean (Figure 2a).

4. Perform MaxEnt 1 deconvolution with the following parameters: Ranges 145000165000, Resolution: 1.00 Da/channel, Uniform Gaussian width at half height: $5 \mathrm{Da}$, and minimum intensity ratios: $33 \%$ left and 33\% right (Note 3 ).

5. Take the intensities of each individual DAR population to assess the DLD (Figure 2b) and calculate the DARav (see section 3.4).

\section{c. Ion mobility mass spectrometry settings for native IM-MS}

1. For IM-MS, in the MassLynx Tune windows set the following parameters: Positive ion mode, $\mathrm{m} / \mathrm{z}$ range from 1000 to 10000 , sampling cone: $80 \mathrm{~V}$ (Note 4), backing pressure: 6 mbar, source offset: $5 \mathrm{~V}$, source temperature: $90^{\circ} \mathrm{C}$, trap collision energy: $4 \mathrm{~V}$, transfer collision energy: $5 \mathrm{~V}$, trap gas: $5 \mathrm{~mL} / \mathrm{min}$, scan time: $4 \mathrm{~s}$, and acquisition time: $2 \mathrm{~min}$.

2. In the Ion mobility cell set the following parameters: $\mathrm{N}_{2}$ flow rate: $45 \mathrm{~mL} / \mathrm{min}$, He flow rate: $120 \mathrm{~mL} / \mathrm{min}$, IM wave height: $40 \mathrm{~V}$, and IM wave velocity $923 \mathrm{~m} / \mathrm{s}$ (Note 5).

3. In the masslynx tune page, activate the Mobility TOF mode in the acquisition mode menu.

4. Deliver among 10-20 $\mu \mathrm{L}$ of $\mathrm{ADC}$ solution at $10 \mu \mathrm{M}$ concentration (Note 6).

5. Record the mass spectrum. Do not forget to activate the option "add drift time function" to record the arrival time distribution of each ion (Note 7).

6. Deliver among $10-20 \mu \mathrm{L}$ of each individual solution of IM calibrants previously prepared. 
7. Record the IM chromatogram of each individual calibrant in triplicate and under identical instrumental conditions as those used for the ADC IM-MS analysis. [Figure 3 near here]

\section{d. Data interpretation Ion Mobility Synapt G2}

1. In the chromatogram menu of MassLynx, open the IM function.

2. Combine the whole mobilogram signal to extract the mass spectrum (Figure 3a).

3. In your mass spectrum, combine the peak of interest at the half-maximum. The chromatogram menu will automatically open, showing the arrival time distribution associated with the ion selected previously (Figure 3b).

4. Take the drift time associated with the center of the ATD Gaussian.

\section{e. Calibration curve to convert drift time on collision cross section (CCS)}

1. Proceed as described in section 3.2.d. to obtain the drift time associated with each peak of the calibrant solutions: For concanavalin A take the drift time of the 19+, 20+, and $21+$ charge states, for ADH take the $24+, 25+$, and $26+$ charge states, and finally for PK take the $32+, 33+$, and $34+$ charge states.

2. Correct the drift times of all these charge states using the following equation (Note 8):

$$
t_{d}^{\prime}=t_{d}-\left(c * \sqrt{\frac{m}{z}}\right) / 1000
$$

3. Calculate the reduced cross section $\left(\Omega^{\prime}\right)$ of the reference charge states of the calibrant systems reported in the literature with the following equation:

$$
\Omega^{\prime}=\left(\Omega_{\text {literature }} \sqrt{\mu}\right) / \mathbf{z}
$$

4. Make a graph $\ln \left(t d^{\prime}\right)$ vs $\ln \left(\Omega^{\prime}\right)$, fit the data with a linear equation

$$
\ln \left(\Omega^{\prime}\right)=a+b \ln \left(t_{d}{ }^{\prime}\right)
$$

5. Make the final correction of the drift times from the equation:

$$
\boldsymbol{t}_{d}^{\prime \prime}=\boldsymbol{t}_{d}^{\prime X}(z \mid \sqrt{\mu})
$$


6. Build a second graph $t_{d}^{\prime \prime} v s \Omega_{\text {literatutre }}$ and fit the data with a linear regression

$$
\Omega_{\text {literature }}=\boldsymbol{b} \boldsymbol{t}_{\boldsymbol{d}}{ }^{\prime \prime}
$$

7. Use the $\mathrm{b}$ and $\mathrm{X}$ parameters to convert the drift time of the different DAR populations of your ADC sample on CCS values (Figure 3c)with the following equation:

$$
\operatorname{CCS}\left(n m^{2}\right)=\left(t_{d}-\left(1.57 \sqrt{\frac{m}{z}}\right) / 1000\right)^{X} Z(1 / \sqrt{\mu}) b
$$

\subsection{Online SEC-native MS}

\section{a. LC setup for SEC-native MS}

2. The column used is a BEH SEC column $200 \AA$, $1.7 \mu \mathrm{m}, 4.6$ x $150 \mathrm{~mm}$ (Note 9). the chromatographic system is an Acquity UPLC H-Class system (Waters, Manchester, UK) comprising a quaternary solvent manager, a sample manager set at $10^{\circ} \mathrm{C}$, a column oven at room temperature and a TUV detector operating at 280 and $214 \mathrm{~nm}$.

3. For the analyses of ADC, the elution is performed in isocratic mode with the following flow rate gradient: $0.25 \mathrm{~mL} / \mathrm{min}$ over $4 \mathrm{~min}$, then $0.1 \mathrm{~mL} / \mathrm{min}$ over $5.5 \mathrm{~min}$ and finally $0.25 \mathrm{~mL} / \mathrm{min}$ over $2.5 \mathrm{~min}$. (Note 10), (Note 11).

4. Load the LC method and equilibrate the LC system with ten column volumes of mobile phase (see 2.1).

\section{b. Native mass spectrometry settings for SEC-native MS experiment}

1. Prior to ADC mass analysis, perform Synapt $\mathrm{G} 2$ calibration with $8 \mu \mathrm{L}$ of cesium iodide solution (see 2.4).

2. For the tune page parameters, the Synapt G2 HDMS is operated in sensitive mode with a capillary voltage of $3.0 \mathrm{kV}$. The sample cone and pressure in the interface region were set to $180 \mathrm{~V}$ for lysine conjugate and $120 \mathrm{~V}$ for cysteine conjugate (Note 2) and 6 mbar, respectively. Source and desolvatation temperature were set to 100 and $450^{\circ} \mathrm{C}$, respectively. Desolvatation and cone gas flows were set at 750 and $60 \mathrm{~L} / \mathrm{hr}$, respectively. 
3. The MS file is setup with the following parameters: Start time: 0 min; End time: $12 \mathrm{~min}$; Polarity: Positive; Analyser Mode: Sensitivity; Dynamic Range: Normal; Sensitivity: Normal; Low Mass: 1000 Da; High Mass: 10000 Da; Scan Time: 1.5 sec (Continuum); No Trap or Transfer CE. Events for flow state are created to direct salts to waste: 0 min Flow State directed to Waste, 4 min Flow State directed to LC, 9 min Flow State directed to Waste.

4. In MassLynx, create a sample list with the MS file, Inlet file, MS tune file. Specify the sample position (Bottle). The injected volume should be comprised between 1 and 10 $\mu \mathrm{L}$. No manual desalting is needed prior SEC-native MS experiment due to online desalting provided by SEC.

\section{c. Data Interpretation}

1. Chromatogram (Figure 4a) and TIC are obtained in the Chromatogram panel.

2. In MaxEnt 1 , the ranges are set from 145000 to 162000 with a resolution of 1 Da/channel. The width at half height is set to 2 Da for the uniform Gaussian. The deconvoluted spectrum is presented in Figure 4b (Note 12).

3. For the DARav calculation, see 3.4. [Figure 4 near here]

\section{d. Ion mobility settings for SEC-native IM-MS experiment}

1. In the MassLynx Tune window set the following parameters: Positive ion mode, $\mathrm{m} / \mathrm{z}$ range from 1000 to 10000 , sampling cone: $80 \mathrm{~V}$, backing pressure: 6 mbar, source offset: $5 \mathrm{~V}$, source temperature: $100^{\circ} \mathrm{C}$, Desolvatation temperature: $450^{\circ} \mathrm{C}$ trap collision energy: $4 \mathrm{~V}$, transfer collision energy: $5 \mathrm{~V}$, trap gas: $5 \mathrm{~mL} / \mathrm{min}$.

2. In the Ion mobility cell set the following parameters: $\mathrm{N}_{2}$ flow rate: $45 \mathrm{~mL} / \mathrm{min}$, He flow rate: $130 \mathrm{~mL} / \mathrm{min}$, IM wave height: $40 \mathrm{~V}$, and IM wave velocity $923 \mathrm{~m} / \mathrm{s}$.

3. The MS file is setup with the following parameters: Start time: 0min; End time: $12 \mathrm{~min}$; Polarity: Positive; Analyser Mode: Sensitivity; Dynamic Range: Normal; Sensitivity: 
Normal; Low Mass: 1000 Da; High Mass: 10000 Da; Scan Time: 1.5 sec (Continuum); No Trap or Transfer CE. Check Add Drift Time Function and Maintain Mobility Separation. Events for flow state are created to direct salts to waste: 0 min Flow State directed to Waste, 4 min Flow State directed to LC, 9 min Flow State directed to Waste.

4. In MassLynx, create a sample list with the MS file, Inlet file, MS tune file. Specify the sample position (Bottle). The injected volume has to be comprised between 1 and 10 $\mu \mathrm{L}$. Record each IM experiment in triplicate to calculate a more reliable collisional cross section.

5. Deliver among 1-10 $\mu \mathrm{L}$ of each individual solution of IM calibrants (see 2.5.2).

6. Record the IM chromatogram of each individual calibrant in triplicate and under identical instrumental conditions as those used for the ADC SEC-native MS analysis.

7. For data interpretation, see 3.2.e

\subsection{Average DAR calculation and Drug Load Distribution}

The average DAR value represents the sum of relative peak intensities in the mass spectrum for each DAR multiplied by its corresponding number of drugs. It was calculated from the relative peak intensities measured from the raw mass spectra, by using the following equation

$$
D A R_{a v}=\frac{\sum_{0}^{8} k \cdot I_{k}}{\sum_{0}^{8} I_{k}}
$$

Where $\mathrm{k}$ is the number of drugs and $\mathrm{I}_{\mathrm{k}}$ is the relative peak intensity of $\mathrm{DAR}_{\mathrm{k}}$.

\section{Notes}

1. Ion mobility calibrants have to be chosen depending on the mass and the charge state of the analyzed $\mathrm{mAb} / \mathrm{ADC}$. Here we chose to use ion mobility calibrants that range from 100 
$\mathrm{kDa}$ to $230 \mathrm{kDa}$ and exhibit positive charge states from $15+$ to $35+$. Usually ADCs are proteins around $150 \mathrm{kDa}$ with $20+$ to $28+$ positive charges under our experimental conditions.

2. The sampling cone $(\mathrm{Vc})$ voltage is a crucial parameter on Waters instrument. A high voltage $(180 \mathrm{~V})$ increases transmission of high $\mathrm{m} / \mathrm{z}$ ions along with more efficient desolvation[14]. However, high Vc values also favor dissociation of noncovalent assemblies, especially for cysteine linked ADCs. An intermediate Vc value of 100-120 V is thus usually more adapted. An optimum value has to be found to provide compromise between good quality MS signal and maintaining ADC integrity.

3. The parameters of the MaxEnt1 deconvolution are protein dependent. In particular, the mass range and the FWHM have to be optimized for each mAb/ADC. The latter has to be chosen as a function of the resolution of the experimental mass spectrum.

4. The sampling cone voltage applied in the source of the mass spectrometer is an important parameter for native IM-MS experiments. Ion heating can occur as a consequence of the effect of this acceleration voltage and eventually can lead to conformational changes (protein unfolding) on protein structures while ions are transmitted into the mass spectrometer. Be sure that the sampling cone voltage you are using allows the transmission of your molecular ions of interest and does not induce any structural changes. From our experience, $80 \mathrm{~V}$ provides a good MS signal (intensity and resolution) without modifying ADCs conformation.

5. These parameters are going to drive the separation of the ions in the ion mobility cell. They have to be carefully optimized to ensure ion focusing (He flow) prior to IM separation and provide the best separation ( $\mathrm{N}_{2}$ flow, wave height, and wave velocity[15]). These parameters are protein dependent. 
6. When performing native IM-MS experiments, we slightly increase the concentration of the analyte compared to native MS experiments to compensate the loss of sensitivity due to the activation of the IM device.

7. Record each IM experiment in triplicate to calculate a more reliable collision cross section.

8. The $c$ constant has been determined empirically and it is specific of the Synapt G2 mass spectrometer. For ion signals lower than $5000 \mathrm{~m} / \mathrm{z}, c=1.41$. For ion signals above 5000 $\mathrm{m} / \mathrm{z}, c=1.57[16]$.

9. Other columns are available for SEC-native MS experiment. For a unique fast desalting goal, shorter column dimension (i.e. BEH SEC 4.6 x 30 mm, $200 \AA$, Waters) could be used. For a separation goal, longer column dimension (i.e. BEH SEC 4.6 x 150/300 mm, $200 \AA$, Waters) is preferred.

10. The flow rate gradient provides improved sensitivity on the Synapt G2 HDMS mass spectrometer.

11. The flow rate gradient method is a generic method which can be easily adapted. If the LC method is changed, do not forget to change events in MS file for directing salts to waste.

12. SEC-native MS coupling provides higher MS spectral resolution and mass accuracy due to the more efficient desalting afforded by SEC compared to manual desalting.

\section{References}

1. Chari RVJ (2008) Targeted cancer therapy: Conferring specificity to cytotoxic drugs. Acc Chem Res 41 (1):98-107. 
2. Carter PJ, Lazar GA (2018) Next generation antibody drugs: pursuit of the 'high-hanging fruit'. Nature Reviews Drug Discovery 17 (3):197-223.

3. Valliere-Douglass JF, McFee WA, Salas-Solano O (2012) Native Intact Mass Determination of Antibodies Conjugated with Monomethyl Auristatin E and F at Interchain Cysteine Residues. Anal Chem 84 (6):2843-2849.

4. Chen J, Yin S, Wu YJ, Ouyang J (2013) Development of a Native Nanoelectrospray Mass Spectrometry Method for Determination of the Drug-to-Antibody Ratio of Antibody-Drug Conjugates. Anal Chem 85 (3):1699-1704.

5. Hengel SM, Sanderson R, Valliere-Douglass J, Nicholas N, Leiske C, Alley SC (2014) Measurement of in Vivo Drug Load Distribution of Cysteine-Linked Antibody-Drug Conjugates Using Microscale Liquid Chromatography Mass Spectrometry. Anal Chem 86 (7):3420-3425.

6. Debaene F, Boeuf A, Wagner-Rousset E, Colas O, Ayoub D, Corvaia N, Van Dorsselaer A, Beck A, Cianferani S (2014) Innovative Native MS Methodologies for Antibody Drug Conjugate Characterization: High Resolution Native MS and IM-MS for Average DAR and DAR Distribution Assessment. Anal Chem 86 (21):10674-10683.

7. Marcoux J, Champion T, Colas O, Wagner-Rousset E, Corvaïa N, Van Dorsselaer A, Beck A, Cianférani S (2015) Native mass spectrometry and ion mobility characterization of trastuzumab emtansine, a lysine-linked antibody drug conjugate. Protein Science : A Publication of the Protein Society 24 (8):1210-1223.

8. Botzanowski T, Erb S, Hernandez-Alba O, Ehkirch A, Colas O, Wagner-Rousset E, Rabuka D, Beck A, Drake PM, Cianférani S (2017) Insights from native mass spectrometry approaches for top- and middle- level characterization of site-specific antibody-drug conjugates. mAbs 9 (5):801-811.

9. Ehkirch A, Hernandez-Alba O, Colas O, Beck A, Guillarme D, Cianférani S (2018) Hyphenation of size exclusion chromatography to native ion mobility mass spectrometry for the analytical characterization of therapeutic antibodies and related products. J Chromatogr B 1086:176-183.

10. Ehkirch A, Goyon A, Hernandez-Alba O, Rouviere F, D'Atri V, Dreyfus C, Haeuw J-F, Diemer H, Beck A, Heinisch S, Guillarme D, Cianferani S (2018) A Novel Online Four-Dimensional SEC $\times$ SEC-IM $\times$ MS Methodology for Characterization of Monoclonal Antibody Size Variants. Anal Chem 90 (23):13929-13937.

11. Ehkirch A, D'Atri V, Rouviere F, Hernandez-Alba O, Goyon A, Colas O, Sarrut M, Beck A, Guillarme D, Heinisch S, Cianferani S (2018) An Online Four-Dimensional HIC $\times$ SEC-IM $\times$ MS Methodology for Proof-of-Concept Characterization of Antibody Drug Conjugates. Anal Chem 90 (3):1578-1586.

12. D'Atri V, Causon T, Hernandez-Alba O, Mutabazi A, Veuthey JL, Cianferani S, Guillarme D (2018) Adding a new separation dimension to MS and LC-MS: What is the utility of ion mobility spectrometry? J Sep Sci 41 (1):20-67.

13. Beck A, D'Atri V, Ehkirch A, Fekete S, Hernandez-Alba O, Gahoual R, Leize-Wagner E, François Y, Guillarme D, Cianférani S (2019) Cutting-edge multi-level analytical and structural characterization of antibody-drug conjugates: present and future. Expert Review of Proteomics:1-26.

14. Ramstrom H, Sanglier S, Leize-Wagner E, Philippe C, Van Dorsselaer A, Haiech J (2003) Properties and regulation of the bifunctional enzyme HPr kinase/phosphatase in Bacillus subtilis. J Biol Chem 278 (2):1174-1185.

15. Atmanene C, Petiot-Becard S, Zeyer D, Van Dorsselaer A, Hannah VV, Sanglier-Cianferani S (2012) Exploring Key Parameters to Detect Subtle Ligand-Induced Protein Conformational Changes Using Traveling Wave Ion Mobility Mass Spectrometry. Anal Chem 84 (11):47034710.

16. Ruotolo BT, Benesch JLP, Sandercock AM, Hyung S-J, Robinson CV (2008) Ion mobilitymass spectrometry analysis of large protein complexes. Nature Protocols 3:1139. 


\section{Figure Captions}

Figure 1. Native MS analysis of deglycosylated brentuximab vedotin (Cysteine conjugate) on Exactive Plus EMR. a) Native mass spectrum corresponding to brentuximab vedotin. b) Deconvoluted mass spectrum representing the drug load distribution with the average DAR.

Figure 2. NanoESI MS analysis in non-denaturing conditions of deglycosylated Inotuzumab Ozogamicin (IO), a lysine ADC, after direct infusion. a) Full scan native mass spectrum of IO representing the different DAR populations (from DAR 2 to DAR 8).

Figure 3. NanoESI IM-MS analysis in non-denaturing conditions of deglycosylated site-specific DAR4 ADC[8] after direct infusion. a) Native mass spectrum of deglycosylated site-specific DAR4 ADC. b) Arrival time distributions (ATDs) of different DAR 4 charge states observed in the mass spectrum. c) Experimental collision cross section $\left({ }^{\mathrm{TW}} \mathrm{CCS}_{\mathrm{N} 2}\right)$ and drift times corresponding to the different charge state populations upon ion mobility separation.

Figure 4. SEC-native MS analysis of deglycosylated inotuzumab ozogamicin (Lysine conjugate). a) SEC-UV chromatogram corresponding to ADC monomer. b) Deconvoluted mass spectrum representing the drug load distribution with the average DAR. 


\section{Figures}
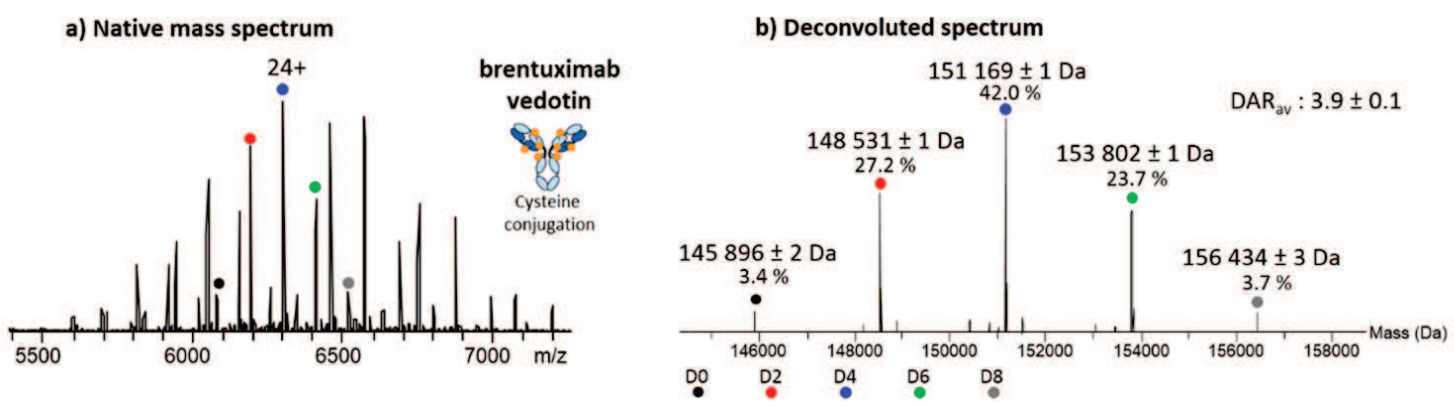

Figure 1. Native MS analysis of deglycosylated brentuximab vedotin (Cysteine conjugate) on Exactive Plus EMR. a) Native mass spectrum corresponding to brentuximab vedotin. b) Deconvoluted mass spectrum representing the drug load distribution with the average DAR.

a) Native mass spectrum

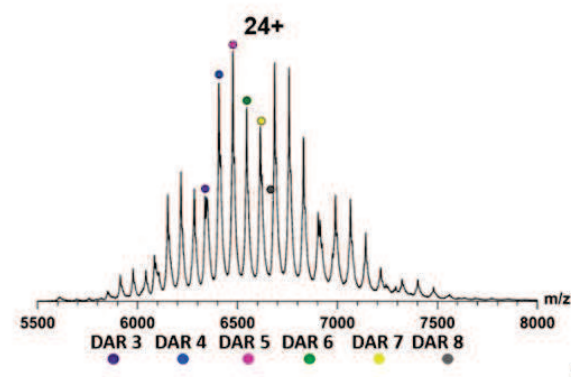

c) Deconvoluted mass spectrum b) Drug load distribution and average DAR

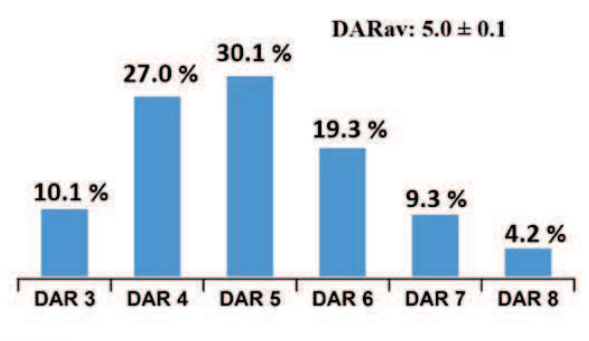

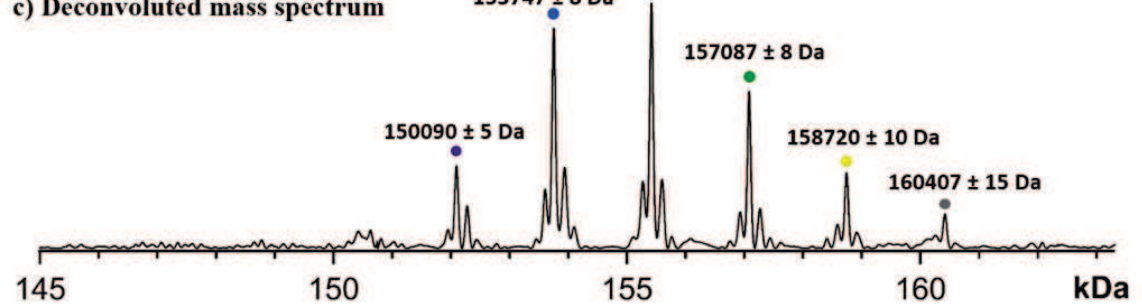

Figure 2. NanoESI MS analysis in non-denaturing conditions of deglycosylated Inotuzumab Ozogamicin (IO), a lysine ADC, after direct infusion. a) Full scan native mass spectrum of IO representing the different DAR populations (from DAR 2 to DAR 8). 
a) Native mass spectrum

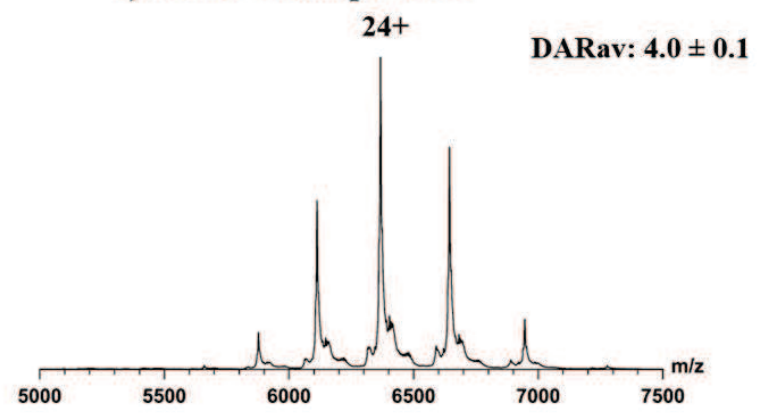

b) Arrival time distributions

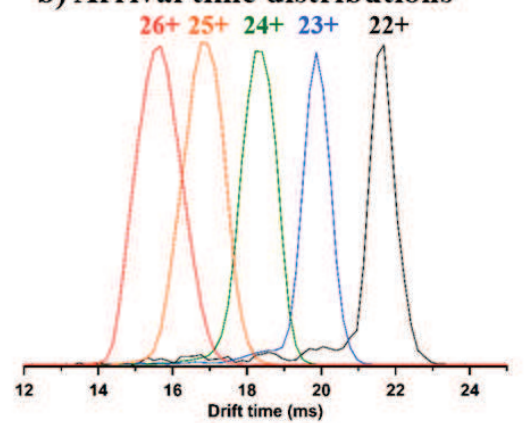

c) Collision cross section and drift times of different DAR4 site specific ADC charge states

\begin{tabular}{c|ccccc}
$\mathrm{Z}$ & $\mathbf{2 2 +}$ & $\mathbf{2 3 +}$ & $\mathbf{2 4 +}$ & $\mathbf{2 5 +}$ & $\mathbf{2 6 +}$ \\
\hline \hline${ }^{\mathrm{TW}} \mathbf{C C S}_{\mathbf{N 2}}\left(\mathbf{n m}^{2}\right)$ & $65.7 \pm 0.1$ & $67.6 \pm 0.1$ & $69.4 \pm 0.1$ & $71.2 \pm 0.1$ & $72.8 \pm 0.1$ \\
Drift time (ms) & $21.7 \pm 0.1$ & $19.9 \pm 0.1$ & $18.4 \pm 0.1$ & $17.0 \pm 0.1$ & $15.6 \pm 0.1$
\end{tabular}

Figure 3. NanoESI IM-MS analysis in non-denaturing conditions of deglycosylated sitespecific DAR4 $\mathrm{ADC}^{8}$ after direct infusion. a) Native mass spectrum of deglycosylated site-specific DAR4 ADC. b) Arrival time distributions (ATDs) of different DAR 4 charge states observed in the mass spectrum. c) Experimental collision cross section $\left({ }^{\mathrm{TW}} \mathrm{CCS}_{\mathrm{N} 2}\right)$ and drift times corresponding to the different charge state populations upon ion mobility separation.
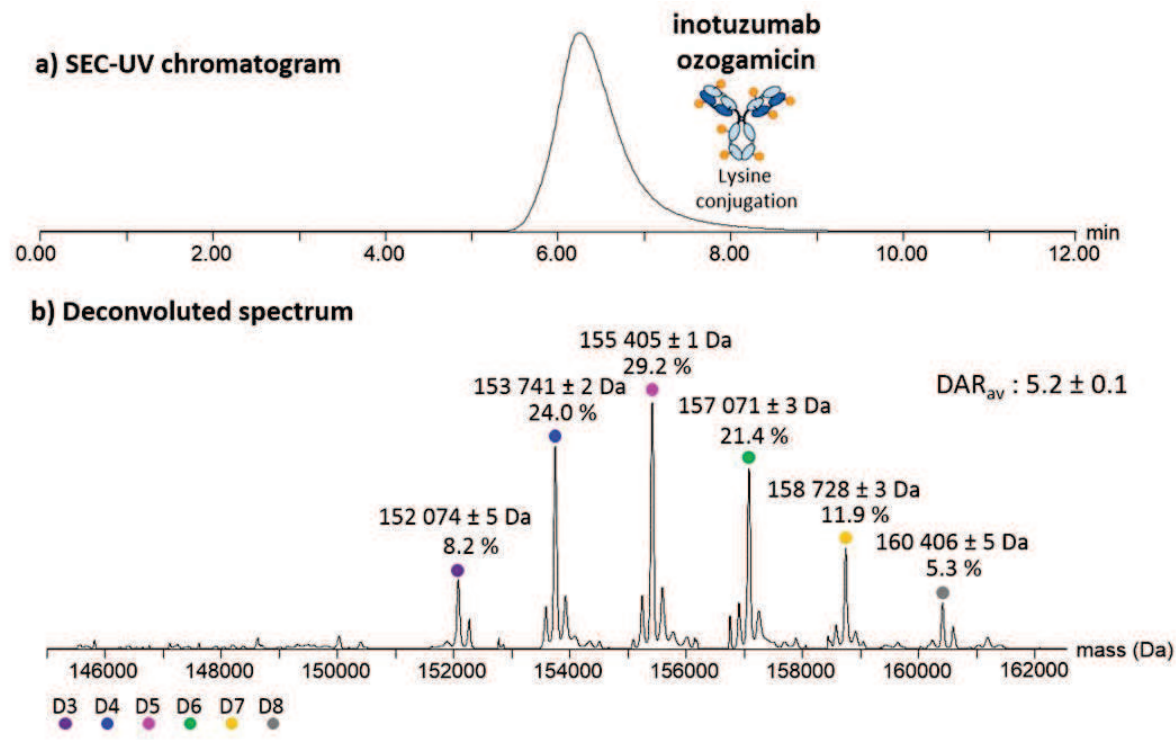

Figure 4. SEC-native MS analysis of deglycosylated inotuzumab ozogamicin (Lysine conjugate). a) SEC-UV chromatogram corresponding to ADC monomer. b) Deconvoluted mass spectrum representing the drug load distribution with the average DAR. 